\title{
Complete Self-Shrinking Solutions for Lagrangian Mean Curvature Flow in Pseudo-Euclidean Space
}

\author{
Ruiwei Xu and Linfen Cao \\ Department of Mathematics, Henan Normal University, Xinxiang, Henan 453007, China \\ Correspondence should be addressed to Linfen Cao; caolf2010@yahoo.com
}

Received 14 January 2014; Accepted 15 June 2014; Published 6 July 2014

Academic Editor: Ljubomir B. Ćirić

Copyright (C) 2014 R. Xu and L. Cao. This is an open access article distributed under the Creative Commons Attribution License, which permits unrestricted use, distribution, and reproduction in any medium, provided the original work is properly cited.

Let $f(x)$ be a smooth strictly convex solution of $\operatorname{det}\left(\partial^{2} f / \partial x_{i} \partial x_{j}\right)=\exp \left\{(1 / 2) \sum_{i=1}^{n} x_{i}\left(\partial f / \partial x_{i}\right)-f\right\}$ defined on a domain $\Omega \subset \mathbb{R}^{n}$; then the graph $M_{\nabla f}$ of $\nabla f$ is a space-like self-shrinker of mean curvature flow in Pseudo-Euclidean space $\mathbb{R}_{n}^{2 n}$ with the indefinite metric $\sum d x_{i} d y_{i}$. In this paper, we prove a Bernstein theorem for complete self-shrinkers. As a corollary, we obtain if the Lagrangian graph $M_{\nabla f}$ is complete in $R_{n}^{2 n}$ and passes through the origin then it is flat.

\section{Introduction}

Let $M$ be an $n$-dimensional submanifold immersed into the Euclidean space $\mathbb{R}^{n+m}$. Mean curvature flow is a oneparameter family $X_{t}=X(\cdot, t)$ of immersions $X_{t}: M \rightarrow$ $\mathbb{R}^{n+m}$ with corresponding images $M_{t}=X_{t}(M)$ such that

$$
\begin{gathered}
\frac{d}{d t} X(x, t)=H(x, t), \quad x \in M, \\
X(x, 0)=X(x)
\end{gathered}
$$

is satisfied, where $H(x, t)$ is the mean curvature vector of $M_{t}$ at $X(x, t)$ in $\mathbb{R}^{n+m}$. Self-similar solutions to the mean curvature flow play an important role in understanding the behavior of the flow and the types of singularities. They satisfy a system of quasilinear elliptic PDE of the second order as follows:

$$
H=-\frac{X^{\perp}}{2}
$$

where $(\cdots)^{\perp}$ stands for the orthogonal projection into the normal bundle $N M$.

Self-shrinkers in the ambient Euclidean space have been studied by many authors; for example, see [1-6] and so forth. For recent progress and related results, see the introduction in [7]. When the ambient space is a pseudo-Euclidean space, there are many classification works about self-shrinkers; for example, see [8-13] and so forth. But very little is known when self-shrinkers are complete not compact with respect to induced metric from pseudo-Euclidean space. In this paper, we will characterize self-shrinkers for Lagrangian mean curvature flow in the pseudo-Euclidean space from this aspect.

Let $\left(x_{1}, \ldots, x_{n} ; y_{1}, \ldots, y_{n}\right)$ be null coordinates in $2 n$ dimensional pseudo-Euclidean space $\mathbb{R}_{n}^{2 n}$. Then, the indefinite metric (cf. [14]) is defined by $d s^{2}=\sum_{i=1}^{n} d x_{i} d y_{i}$. Suppose $f(x)$ is a smooth strictly convex function defined on domain $\Omega \subset \mathbb{R}^{n}$. The graph $M_{\nabla f}$ of $\nabla f$ can be written as $\left(x_{1}, \ldots, x_{n} ; \partial f / \partial x_{1}, \ldots, \partial f / \partial x_{n}\right)$. Then, the induced Riemannian metric on $M_{\nabla f}$ is given by

$$
G=\sum_{i=1}^{n} \frac{\partial^{2} f}{\partial x_{i} \partial x_{j}} d x_{i} d x_{j} .
$$

In particular, if function $f$ satisfies

$$
\operatorname{det}\left(\frac{\partial^{2} f}{\partial x_{i} \partial x_{j}}\right)=\exp \left\{\frac{1}{2} \sum_{i=1}^{n} x_{i} \frac{\partial f}{\partial x_{i}}-f\right\},
$$

then the graph $M_{\nabla f}$ of $\nabla f$ is a space-like self-shrinking solution for mean curvature flow in $\mathbb{R}_{n}^{2 n}$.

Huang and Wang [12] and Chau et al. [8] have used different methods to investigate the entire solutions to the 
above equation and showed that an entire smooth strictly convex solution to (4) in $\mathbb{R}^{n}$ is the quadratic polynomial under the decay condition on Hessian of $f$. Later Ding and Xin in $[10]$ improve the previous ones in $[8,12]$ by removing the additional assumption and prove the following.

Theorem 1. Any space-like entire graphic self-shrinking solution to Lagrangian mean curvature flow in $R_{n}^{2 n}$ with the indefinite metric $\sum_{i} d x_{i} d y_{i}$ is flat.

These rigidity results assume that the self-shrinker graphs are entire. Namely, they are Euclidean complete. Here, we will characterize the rigidity of self-shrinker graphs from another completeness and pose the following problem.

If a graphic self-shrinker is complete with respect to induced metric from ambient space $R_{n}^{2 n}$, then is it flat?

In this paper, we will use affine technique (see [15-18]) to prove the following Bernstein theorem. As a corollary, it gives a partial affirmative answer to the above problem.

Theorem 2. Let $f(x)$ be a $C^{\infty}$ strictly convex function defined on a convex domain $\Omega \subseteq \mathbb{R}^{n}$ satisfying the PDE (4). If there is a positive constant $\alpha$ depending only on $n$ such that the hypersurface $M=\{(x, f(x))\}$ in $\mathbb{R}^{n+1}$ is complete with respect to the metric

$$
\widetilde{G}=\exp \left\{\alpha \sum x_{i} \frac{\partial f}{\partial x_{i}}\right\} \sum \frac{\partial^{2} f}{\partial x_{i} \partial x_{j}} d x_{i} d x_{j}
$$

then $f$ is the quadratic polynomial.

Remark 3. If $f(x)$ is a strictly convex solution to (4), then the graph $\{(x, \nabla f / 2 n \alpha)\}$ is a minimal manifold in $R_{n}^{2 n}$ endowed with the conformal metric $d s^{2}=\exp \{-\alpha x \cdot y\} d x \cdot d y$. ing.

As a direct application of Theorem 2, we have the follow-

Corollary 4. Let $f$ be a strictly convex $C^{\infty}$-function defined on a convex domain $\Omega \subset \mathbb{R}^{n}$. If the graph $M_{\nabla f}=\{(x, \nabla f(x))\}$ in $\mathbb{R}_{n}^{2 n}$ is a complete space-like self-shrinker for mean curvature flow and the sum $\sum x_{i}\left(\partial f / \partial x_{i}\right)$ has a lower bound, then $M_{\nabla f}$ is flat.

When the shrinker passes through the origin especially, we have the following corollary.

Corollary 5. If the graph $M_{\nabla f}=\{(x, \nabla f(x))\}$ in $\mathbb{R}_{n}^{2 n}$ is a complete space-like self-shrinker for mean curvature flow and passes through the origin, then $M_{\nabla f}$ is flat.

\section{Preliminaries}

Let $f\left(x_{1}, \ldots, x_{n}\right)$ be a strictly convex $C^{\infty}$-function defined on a domain $\Omega \subset \mathbb{R}^{n}$. Consider the graph hypersurface

$$
M:=\left\{(x, f(x)) \mid x_{n+1}=f\left(x_{1}, \ldots, x_{n}\right),\left(x_{1}, \ldots, x_{n}\right) \in \Omega\right\}
$$

For $M$, we choose the canonical relative normalization $Y=$ $(0,0, \ldots, 1)$. Then, in terms of the language of the relative affine differential geometry, the Calabi metric

$$
G=\sum f_{i j} d x_{i} d x_{j}
$$

is the relative metric with respect to the normalization $Y$. For the position vector $y=\left(x_{1}, \ldots, x_{n}, f\left(x_{1}, \ldots, x_{n}\right)\right)$, we have

$$
y_{, i j}=\sum A_{i j}^{k} y_{k}+f_{i j} Y
$$

where "', denotes the covariant derivative with respect to the Calabi metric $G$. We recall some fundamental formulas for the graph $M$; for details, see [19]. The Levi-Civita connection with respect to the metric $G$ has the Christoffel symbols

$$
\Gamma_{i j}^{k}=\frac{1}{2} \sum f^{k l} f_{i j l} .
$$

The Fubini-Pick tensor $A_{i j k}$ satisfies

$$
A_{i j k}=-\frac{1}{2} f_{i j k}
$$

Consequently, for the relative Pick invariant, we have

$$
J=\frac{1}{4 n(n-1)} \sum f^{i l} f^{j m} f^{k n} f_{i j k} f_{l m n} .
$$

The Gauss integrability conditions and the Codazzi equations read

$$
\begin{gathered}
R_{i j k l}=\sum f^{m h}\left(A_{j k m} A_{h i l}-A_{i k m} A_{h j l}\right), \\
A_{i j k, l}=A_{i j l, k} .
\end{gathered}
$$

From (12), we get the Ricci tensor

$$
R_{i k}=\sum f^{m h} f^{l j}\left(A_{i m l} A_{h j k}-A_{i m k} A_{h l j}\right) .
$$

Introduce the Legendre transformation of $f$

$$
\begin{gathered}
\xi_{i}=\frac{\partial f}{\partial x_{i}}, \quad i=1,2, \ldots, n, \\
u\left(\xi_{1}, \ldots, \xi_{n}\right)=\sum_{i=1}^{n} x_{i} \frac{\partial f}{\partial x_{i}}-f(x) .
\end{gathered}
$$

Define the functions

$$
\begin{gathered}
\rho:=\left[\operatorname{det}\left(\frac{\partial^{2} f}{\partial x_{i} \partial x_{j}}\right)\right]^{-1 /(n+2)}=\left[\operatorname{det}\left(\frac{\partial^{2} u}{\partial \xi_{i} \partial \xi_{j}}\right)\right]^{1 /(n+2)}, \\
\Phi:=\sum f^{i j}(\ln \rho)_{i}(\ln \rho)_{j}=\frac{\|\nabla \rho\|^{2}}{\rho^{2}},
\end{gathered}
$$

here and later the norm $\|\cdot\|$ is defined with respect to the Calabi metric. From the PDE (4), we obtain

$$
\frac{\partial \ln \rho}{\partial x_{i}}=\frac{1}{2(n+2)}(f-u)_{i}=\frac{1}{2(n+2)}\left\{f_{i}-x_{k} f_{k i}\right\} \text {. }
$$


That is,

$$
x_{i}=\sum f^{i k}\left(f_{k}-2(n+2)(\ln \rho)_{k}\right) \text {. }
$$

Using (17) and (18), we can get

$$
\rho_{i j}=\frac{\rho_{i} \rho_{j}}{\rho}+f^{k l} f_{i j k} \rho_{l}-\frac{f^{k l} f_{i j k} f_{l} \rho}{2(n+2)} .
$$

Put $\tau:=(1 / 2) \sum f^{i j}\left(\rho_{i} / \rho\right) f_{j}$. From (19), we have

$$
\Delta \rho=-\frac{n}{2} \frac{\|\nabla \rho\|^{2}}{\rho}+\tau \rho .
$$

By (17), we get

$$
4(n+2)^{2} \Phi=\|\nabla u\|^{2}+\|\nabla f\|^{2}-2(f+u),
$$

and then

$$
\|\nabla(f+u)\|^{2}=4(n+2)^{2} \Phi+4(f+u) .
$$

Using (17) yields

$$
\Delta(f+u)=2 n+(n+2)^{2} \Phi .
$$

Define a conformal Riemannian metric $\widetilde{G}:=\exp \{\alpha(f+u)\} G$, where $\alpha$ is a constant.

Conformal Ricci Curvature. Denote by $\widetilde{R}_{i j}$ the Ricci curvature with respect to the metric $\widetilde{G}$; then

$$
\begin{aligned}
\widetilde{R}_{i j}= & R_{i j}-\frac{(n-2) \alpha}{2}(f+u)_{, i j}+\frac{(n-2) \alpha^{2}}{4}(f+u)_{, i}(f+u)_{, j} \\
& -\frac{1}{2}\left(\alpha \Delta(f+u)+\frac{(n-2) \alpha^{2}}{2}\|\nabla(f+u)\|^{2}\right) G_{i j},
\end{aligned}
$$

where "', again denotes the covariant derivation with respect to the Calabi metric.

Using the above formulas, we can get the following crucial estimates.

Proposition 6. Let $f\left(x_{1}, \ldots, x_{n}\right)$ be a $C^{\infty}$ strictly convex function satisfying PDE (4). Then, the following estimate holds:

$$
\begin{aligned}
\Delta \Phi \geq & A_{1}\langle\nabla \Phi, \nabla \ln \rho\rangle+\frac{1}{4}\langle\nabla(f+u), \nabla \Phi\rangle-A_{2} \frac{\|\nabla \Phi\|^{2}}{\Phi} \\
& +A_{3} \Phi^{2}+\Phi
\end{aligned}
$$

where

$$
\begin{gathered}
A_{1}=\frac{6 n^{2}-n+16}{(n-1)(3 n+4)}, \quad A_{2}=\frac{3 n^{2}+32 n}{8(n-1)(3 n+4)}, \\
A_{3}=\frac{64 n^{3}-72 n^{2}-46 n-72}{5 n(n-1)(3 n+4)} .
\end{gathered}
$$

Because its calculation is standard as in [16], we will give its proof in the appendix.

For affine hyperspheres, Calabi in [20] calculated the Laplacian of the Pick invariant $J$. Later, for a general convex function, $\mathrm{Li}$ and $\mathrm{Xu}$ proved the following lemma in [17].

Lemma 7. The Laplacian of the relative Pick invariant $J$ satisfies

$$
\begin{aligned}
\Delta J \geq & \frac{n+2}{n(n-1)} \sum f^{i l} f^{j m} f^{k n} A_{i j k}(\ln \rho)_{l m n} \\
& +\frac{2}{n(n-1)}\|\nabla A\|^{2}+2 J^{2}-\frac{(n+2)^{4}}{4} \Phi^{2},
\end{aligned}
$$

where "', denotes the covariant derivative with respect to the Calabi metric.

Using Lemma 7, we get the following corollary. For the proof, see the appendix.

Corollary 8. Let $f\left(x_{1}, \ldots, x_{n}\right)$ be a $C^{\infty}$ strictly convex function satisfying $P D E(4)$; then

$$
\begin{gathered}
\Delta J \geq J^{2}-20(n+2)^{8} \Phi^{2}+\frac{1}{4}\langle\nabla J, \nabla(f+u)\rangle \\
+J-\sqrt{n(n-1)}\|\nabla(f+u)\| J^{3 / 2} .
\end{gathered}
$$

\section{Proof of Theorem 2}

It is our aim to prove $\Phi \equiv 0$; thus, from definition of $\rho$,

$$
\operatorname{det}\left(f_{i j}\right)=\text { const. }
$$

everywhere on $M$. As in [8], by Euler homogeneous theorem, we get Theorem 2 .

Denote by $s\left(p_{0}, p\right)$ the geodesic distance function from $p_{0} \in M$ with respect to the metric $\widetilde{G}$. For any positive number $a$, let $B_{a}\left(p_{0}, \widetilde{G}\right):=\left\{p \in M \mid s\left(p_{0}, p\right) \leq a\right\}$. Denote

$$
\begin{aligned}
& \mathscr{A}:=\max _{B_{a}\left(p_{0}, \widetilde{G}\right)}\left\{\left(a^{2}-s^{2}\right)^{2} \exp \{-\alpha(f+u)\} \Phi\right\}, \\
& \mathscr{B}:=\max _{B_{a}\left(p_{0}, \widetilde{G}\right)}\left\{\left(a^{2}-s^{2}\right)^{2} \exp \{-\alpha(f+u)\} J\right\} .
\end{aligned}
$$

Lemma 9. Let $f$ be a strictly convex $C^{\infty}$-function satisfying the PDE (4). Then, there exist positive constants $\alpha$ and $C$, depending only on $n$, such that

$$
\mathscr{A} \leq C\left(a^{2}+a^{3}\right) .
$$

Proof. Step 1. We will prove that there exists a constant $C$ depending only on $n$ such that

$$
\mathscr{A} \leq C\left(\mathscr{B}^{1 / 2} a+a^{2}+a^{3}\right) .
$$

To this end, consider the function

$$
F:=\left(a^{2}-s^{2}\right)^{2} \exp \{-\alpha(f+u)\} \Phi
$$


defined on $B_{a}\left(p_{0}, \widetilde{G}\right)$, where $\alpha$ is a positive constant to be determined later. Obviously, $F$ attains its supremum at some interior point $p^{*}$. We may assume that $s^{2}$ is a $C^{2}$-function in a neighborhood of $p^{*}$. Choose an orthonormal frame field on $M$ around $p^{*}$ with respect to the Calabi metric G. Then, at $p^{*}$,

$$
\begin{gathered}
\frac{\Phi_{, i}}{\Phi}-\alpha(f+u)_{, i}-\frac{4 s s_{, i}}{a^{2}-s^{2}}=0 \\
\frac{\Delta \Phi}{\Phi}-\frac{\sum\left(\Phi_{, i}\right)^{2}}{\Phi^{2}}-\alpha \Delta(f+u)-\frac{12 a^{2} \exp \{\alpha(f+u)\}}{\left(a^{2}-s^{2}\right)^{2}} \\
-\frac{4 s \Delta s}{a^{2}-s^{2}} \leq 0
\end{gathered}
$$

where "', denotes the covariant derivative with respect to the Calabi metric $G$ as before, and we used the fact $\|\nabla s\|_{G}^{2}=$ $\exp \{\alpha(f+u)\}$. Inserting Proposition 6 into (35), we get

$$
\begin{gathered}
-\left(1+A_{2}\right) \frac{\sum\left(\Phi_{, i}\right)^{2}}{\Phi^{2}}+A_{3} \Phi+\frac{1}{4}(f+u)_{, i} \frac{\Phi_{, i}}{\Phi} \\
+A_{1} \frac{\Phi_{, i}}{\Phi} \frac{\rho_{, i}}{\rho}+1-\alpha\left(2 n+(n+2)^{2} \Phi\right) \\
-\frac{12 a^{2} \exp \{\alpha(f+u)\}}{\left(a^{2}-s^{2}\right)^{2}}-\frac{4 s \Delta s}{a^{2}-s^{2}} \leq 0 .
\end{gathered}
$$

Combining (34) with (36) and using the Schwarz inequality, we have

$$
\begin{gathered}
\frac{1}{4} \sum(f+u)_{, i} \frac{\Phi_{, i}}{\Phi} \\
\geq \frac{1}{8} \alpha \sum\left[(f+u)_{, i}\right]^{2}-\frac{2}{\alpha} \frac{a^{2} \exp \{\alpha(f+u)\}}{\left(a^{2}-s^{2}\right)^{2}}, \\
A_{1} \sum \frac{\Phi_{, i}}{\Phi} \frac{\rho_{, i}}{\rho} \geq-\frac{A_{3}}{4} \Phi-\frac{A_{1}^{2}}{A_{3}} \frac{\Phi_{, i}^{2}}{\Phi^{2}} \\
\frac{\sum\left(\Phi_{, i}\right)^{2}}{\Phi^{2}} \leq 2\left(\alpha^{2} \sum\left[(f+u)_{, i}\right]^{2}+16 \frac{a^{2} \exp \{\alpha(f+u)\}}{\left(a^{2}-s^{2}\right)^{2}}\right) .
\end{gathered}
$$

Choose $\alpha$ small enough such that

$$
\begin{gathered}
2\left(1+A_{2}+\frac{A_{1}^{2}}{A_{3}}\right) \alpha \leq \frac{1}{16}, \quad \alpha(n+2)^{2} \leq \frac{A_{3}}{4}, \\
100 n \alpha \leq 1 .
\end{gathered}
$$

Then, by substituting the three estimates above, we get

$$
\begin{aligned}
& \frac{A_{3}}{2} \Phi+\frac{1}{16} \alpha(f+u)_{, i}^{2}-\exp \{\alpha(f+u)\} \frac{C a^{2}}{\left(a^{2}-s^{2}\right)^{2}} \\
& -\frac{4 s \Delta s}{a^{2}-s^{2}} \leq 0
\end{aligned}
$$

here and later $C$ denotes positive constant depending only on $n$.

Denote $a^{*}=s\left(p_{0}, p^{*}\right)$. If $a^{*}=0$, from (39), it is easy to complete the proof of the lemma. In the following, we assume that $a^{*}>0$. Now, we calculate the term $4 s \Delta s /\left(a^{2}-s^{2}\right)$. Firstly, we will give a lower bound of the $\operatorname{Ricci} \operatorname{curvature} \operatorname{Ric}(M, \widetilde{G})$. Assume that

$$
\begin{aligned}
& \max _{B_{a^{*}}\left(p_{0}, \widetilde{G}\right)}\{\exp \{-\alpha(f+u)\} \Phi\}=\exp \{-\alpha(f+u)\} \Phi(\widetilde{p}), \\
& \max _{B_{a^{*}}\left(p_{0}, \widetilde{G}\right)}\{\exp \{-\alpha(f+u)\} J\}=\exp \{-\alpha(f+u)\} J(\widetilde{q}) .
\end{aligned}
$$

For any $p \in B_{a^{*}}\left(p_{0}, \widetilde{G}\right)$, by a coordinate transformation, $f_{i j}(p)=\delta_{i j}$ and $R_{i j}(p)=0$ hold for $i \neq j$. Then, at $p$,

$$
\begin{gathered}
R_{i i} \geq \frac{1}{4}\left(\sum_{m} f_{m i i}^{2}+(n+2) \sum_{m} f_{m i i} \frac{\partial}{\partial x_{m}} \ln \rho\right) \geq-\frac{(n+2)^{2}}{16} \Phi, \\
\frac{(n-2) \alpha}{2}(f+u)_{, i i} \\
=\frac{(n-2) \alpha}{2}\left(2-\frac{1}{2} f_{i i k}(f+u)_{k}\right) \\
\leq(n-2) \alpha+\frac{(n-2) \alpha^{2}}{4}\|\nabla(f+u)\|^{2}+C J .
\end{gathered}
$$

Then, using the Schwarz inequality and (22)-(24), we know that at the point $p$

$$
\begin{aligned}
& \operatorname{Ric}(M, \widetilde{G}) \\
& \quad \geq-\exp \{-\alpha(f+u)\} \\
& \quad \times\{C \Phi+C J+\alpha[3(n-2) \alpha(f+u)+2(n-1)]\} \widetilde{G} . \\
& \text { If } 3(n-2) \alpha(f+u)+2(n-1) \leq 0 \text {, then } \\
& -\exp \{-\alpha(f+u)\} \alpha[3(n-2) \alpha(f+u)+2(n-1)] \geq 0 .
\end{aligned}
$$

Otherwise,

$$
\exp \{-\alpha(f+u)\} \alpha[3(n-2) \alpha(f+u)+2(n-1)] \leq C .
$$

Then, the Ricci curvature $\operatorname{Ric}(M, \widetilde{G})$ on $B_{a^{*}}\left(p_{0}, \widetilde{G}\right)$ is bounded from below by

$$
\begin{aligned}
& \operatorname{Ric}(M, \widetilde{G}) \\
& \quad \geq-C\left(\frac{\Phi}{\exp \{\alpha(f+u)\}}(\widetilde{p})+\frac{J}{\exp \{\alpha(f+u)\}}(\widetilde{q})+1\right) \widetilde{G} .
\end{aligned}
$$


By the Laplacian comparison theorem, we get

$$
\begin{aligned}
& \frac{s \Delta s}{a^{2}-s^{2}} \\
& =\exp \{\alpha(f+u)\} \frac{s \widetilde{\Delta} s}{a^{2}-s^{2}}-\frac{(n-2) \alpha}{2} \frac{s(f+u)_{, i} s_{, i}}{a^{2}-s^{2}} \\
& \leq C_{3} \frac{\exp \{\alpha(f+u)\}\left(p^{*}\right)}{a^{2}-s^{2}} \\
& \quad \times(\sqrt{\exp \{-\alpha(f+u)\} \Phi(\tilde{p})} \\
& +\sqrt{\exp \{-\alpha(f+u)\} J(\widetilde{q})}+1) s \\
& +\frac{\alpha}{16}(f+u)_{, i}^{2}+C \frac{a^{2} \exp \{\alpha(f+u)\}}{\left(a^{2}-s^{2}\right)^{2}}
\end{aligned}
$$

where $\widetilde{\Delta}$ denotes the Laplacian with respect to the metric $\widetilde{G}$.

Substituting (46) into (39) yields

$$
\begin{aligned}
& \exp \{-\alpha(f+u)\} \Phi \\
& \leq a C\left(\sqrt{\frac{\Phi}{\exp \{\alpha(f+u)\}}(\tilde{p})}\right. \\
& \left.+\sqrt{\frac{J}{\exp \{\alpha(f+u)\}}(\tilde{q})}+1\right) \times\left(a^{2}-s^{2}\right)^{-1} \\
& +\frac{C a^{2}}{\left(a^{2}-s^{2}\right)^{2}} .
\end{aligned}
$$

Note that

$$
\begin{aligned}
\mathscr{A} & \geq\left[\left(a^{2}-s^{2}\right)^{2} \exp \{-\alpha(f+u)\} \Phi\right](\tilde{p}) \\
& \geq\left(a^{2}-s^{2}\right)^{2}\left(p^{*}\right) \exp \{-\alpha(f+u)\}(\widetilde{p}) \Phi(\widetilde{p}), \\
\mathscr{B} & \geq\left[\left(a^{2}-s^{2}\right)^{2} \exp \{-\alpha(f+u)\} J\right](\widetilde{q}) \\
& \geq\left(a^{2}-s^{2}\right)^{2}\left(p^{*}\right) \exp \{-\alpha(f+u)\}(\tilde{q}) J(\tilde{q}) .
\end{aligned}
$$

Multiplying by $\left(a^{2}-s^{2}\right)^{2}\left(p^{*}\right)$, at both sides of (47), yields

$$
\mathscr{A} \leq C a\left(\mathscr{A}^{1 / 2}+\mathscr{B}^{1 / 2}\right)+C\left(a^{2}+a^{3}\right)
$$

Using the Schwarz inequality, we complete Step 1.

Step 2. We will prove that there is a constant $C$ depending only on $n$ such that

$$
\mathscr{B} \leq C\left(\mathscr{A}+a^{2}+a^{4}\right)
$$

Consider

$$
H=\left(a^{2}-s^{2}\right)^{2} \exp \{-\alpha(f+u)\} J
$$

defined on $B_{a}\left(p_{0}, \widetilde{G}\right)$, where $\alpha$ is the constant in (38). Obviously, $H$ attains its supremum at some interior point $q^{*}$. Choose an orthonormal frame field on $M$ around $q^{*}$ with respect to the Calabi metric $G$. Then, at $q^{*}$,

$$
\begin{gathered}
\frac{J_{, i}}{J}-\alpha(f+u)_{, i}-\frac{4 s s_{, i}}{a^{2}-s^{2}}=0, \\
\frac{\Delta J}{J}-\frac{\sum\left(J_{, i}\right)^{2}}{J^{2}}-\alpha \Delta(f+u)-\frac{12 a^{2} \exp \{\alpha(f+u)\}}{\left(a^{2}-s^{2}\right)^{2}} \\
-\frac{4 s \Delta s}{a^{2}-s^{2}} \leq 0,
\end{gathered}
$$

where "', denotes the covariant derivative with respect to the Calabi metric $G$ as before. Inserting Corollary 8 into (53), we get

$$
\begin{aligned}
J- & 20(n+2)^{8} \frac{\Phi^{2}}{J}+\frac{1}{4} \sum \frac{J_{, i}}{J}(f+u)_{, i}+1-\frac{\sum\left(J_{, i}\right)^{2}}{J^{2}} \\
- & \alpha\left(2 n+(n+2)^{2} \Phi\right)-\sqrt{n(n-1)}\|\nabla(f+u)\| J^{1 / 2} \\
& -\frac{12 a^{2} \exp \{\alpha(f+u)\}}{\left(a^{2}-s^{2}\right)^{2}}-\frac{4 s \Delta s}{a^{2}-s^{2}} \leq 0 .
\end{aligned}
$$

Applying the Schwarz inequality, we have

$$
\begin{gathered}
\frac{1}{4} \sum \frac{J_{, i}}{J}(f+u)_{, i} \geq \frac{\alpha}{8} \sum\left[(f+u)_{, i}\right]^{2}-C \frac{a^{2} \exp \{\alpha(f+u)\}}{\left(a^{2}-s^{2}\right)^{2}}, \\
\sum \frac{\left(J_{, i}\right)^{2}}{J^{2}} \leq 2 \alpha^{2} \sum\left[(f+u)_{, i}\right]^{2}+\frac{32 a^{2} \exp \{\alpha(f+u)\}}{\left(a^{2}-s^{2}\right)^{2}}, \\
\sqrt{n(n-1)}\|\nabla(f+u)\| J^{1 / 2} \\
\leq \frac{J}{4}+4 n(n-1)\left((n+2)^{2} \Phi+(f+u)\right) .
\end{gathered}
$$

Inserting these estimates into (54) yields

$$
\begin{aligned}
& \frac{3}{4} J-20(n+2)^{8} \frac{\Phi^{2}}{J}-C \Phi+\frac{\alpha}{16} \sum(f+u)_{, i}^{2} \\
& -C \frac{a^{2} \exp \{\alpha(f+u)\}}{\left(a^{2}-s^{2}\right)^{2}}-C(f+u)-\frac{4 s \Delta s}{a^{2}-s^{2}} \leq 0,
\end{aligned}
$$

here and later $C$ denotes different positive constants depending only on $n$.

We discuss two subcases.

Case 1. If

$$
\frac{J}{\exp \{\alpha(f+u)\}}\left(q^{*}\right) \leq \frac{\Phi}{\exp \{\alpha(f+u)\}}\left(q^{*}\right),
$$

then $\mathscr{B} \leq \mathscr{A}$. In this case, Step 2 is complete.

Case 2. Now, assume that

$$
\frac{J}{\exp \{\alpha(f+u)\}}\left(q^{*}\right)>\frac{\Phi}{\exp \{\alpha(f+u)\}}\left(q^{*}\right) \text {. }
$$


Then, $1>(\Phi / J)\left(q^{*}\right)$. Thus,

$$
\begin{aligned}
& \frac{3}{4} J-C \Phi+\frac{\alpha}{16} \sum\left[(f+u)_{, i}\right]^{2}-C \frac{a^{2} \exp \{\alpha(f+u)\}}{\left(a^{2}-s^{2}\right)^{2}} \\
& -C(f+u)-\frac{4 s \Delta s}{a^{2}-s^{2}} \leq 0 .
\end{aligned}
$$

The rest of the estimate is almost the same as in Step 1. The only difference is to deal with the term $(f+u)$. If $(f+u)\left(q^{*}\right) \leq$ 0 , then $-C(f+u)\left(q^{*}\right) \geq 0$. We can drop this term.

Otherwise, $\exp \{-\alpha(f+u)\}(f+u)$ has a uniform upper bound.

Using the same method as in Step 1, we can estimate the term $4 s \Delta s /\left(a^{2}-s^{2}\right)$ and finally get

$$
\mathscr{B} \leq C\left(\mathscr{A}+a^{2}+a^{4}\right) .
$$

Then, combining the conclusion of Step 1, we get

$$
\mathscr{A} \leq C\left(a^{2}+a^{3}\right) \text {. }
$$

This completes the proof of Lemma 9.

Proof of Theorem 2. For any point $q \in M$, choose sufficient large constant $R_{0}$ such that $q \in B_{R_{0}}\left(p_{0}, \widetilde{G}\right)$. Then, for all $a \geq$ $R_{0}, q \in B_{a}\left(p_{0}\right)$. Using Lemma 9 , we know

$$
\exp \{-\alpha(f+u)\} \Phi(q) \leq \frac{C(n)\left(a^{2}+a^{3}\right)}{\left(a^{2}-s^{2}\right)^{2}}
$$

Now, let $a \rightarrow+\infty$, and we have

$$
0 \leq \exp \{-\alpha(f+u)\} \Phi(q) \leq 0 .
$$

Consequently,

$$
\operatorname{det}\left(\frac{\partial^{2} f}{\partial x_{i} \partial x_{j}}\right)(q)=\text { const. }
$$

This completes the proof of Theorem 2 .

\section{Appendix}

Proof of Proposition 6. Let $p \in M$, and we choose a local orthonormal frame field of the metric $G$ around $p$. Then,

$$
\begin{gathered}
\Phi=\frac{\sum\left(\rho_{, j}\right)^{2}}{\rho^{2}}, \quad \Phi_{, i}=2 \sum \frac{\rho_{, j} \rho_{, j i}}{\rho^{2}}-2 \rho_{, i} \frac{\sum\left(\rho_{, j}\right)^{2}}{\rho^{3}} \\
\Delta \Phi=2 \frac{\sum\left(\rho_{, j i}\right)^{2}}{\rho^{2}}+2 \sum \frac{\rho_{, j} \rho_{, j i i}}{\rho^{2}}-8 \sum \frac{\rho_{, j} \rho_{, i} \rho_{, j i}}{\rho^{3}} \\
+(n+6) \Phi^{2}-2 \tau \Phi,
\end{gathered}
$$

where we used (20). In the case $\Phi(p)=0$, it is easy to get, at $p$,

$$
\Delta \Phi \geq 2 \frac{\sum\left(\rho_{, i j}\right)^{2}}{\rho^{2}}
$$

Now, we assume that $\Phi(p) \neq 0$. Choose a local orthonormal frame field of the metric $G$ around $p$ such that $\rho_{, 1}(p)=$ $\|\nabla \rho\|(p)>0, \rho_{, i}(p)=0$, for all $i>1$. Then,

$$
\begin{aligned}
\Delta \Phi= & 2(1-\delta+\delta) \sum \frac{\left(\rho_{, i j}\right)^{2}}{\rho^{2}}+2 \sum \frac{\rho_{, j} \rho_{, j i i}}{\rho^{2}} \\
& -8 \frac{\left(\rho_{, 1}\right)^{2} \rho_{, 11}}{\rho^{3}}+(n+6) \Phi^{2}-2 \tau \Phi,
\end{aligned}
$$

where $1>\delta>0$ is a constant to be determined later. Applying (20), we obtain

$$
\begin{aligned}
2 \frac{\sum\left(\rho_{, i j}\right)^{2}}{\rho^{2}} \geq & \frac{2 n}{n-1} \frac{\left(\rho_{, 11}\right)^{2}}{\rho^{2}}+4 \frac{\sum_{i>1}\left(\rho_{, 1 i}\right)^{2}}{\rho^{2}} \\
& +\frac{2 n}{n-1} \frac{\left(\rho_{, 1}\right)^{2} \rho_{, 11}}{\rho^{3}}+\frac{n^{2}}{2(n-1)} \Phi^{2} \\
& -\frac{4}{n-1} \frac{\rho_{, 11}}{\rho} \tau+\frac{2}{n-1} \tau^{2}-\frac{2 n}{n-1} \Phi \tau .
\end{aligned}
$$

An application of the Ricci identity shows that

$$
\begin{aligned}
\frac{2}{\rho^{2}} \sum \rho_{, j} \rho_{, j i i}= & -2 n \frac{\left(\rho_{, 1}\right)^{2} \rho_{, 11}}{\rho^{3}}+n \frac{\left(\rho_{, 1}\right)^{4}}{\rho^{4}} \\
& +2 R_{11} \frac{\left(\rho_{, 1}\right)^{2}}{\rho^{2}}+2 \frac{\rho_{, 1}}{\rho^{2}}(\rho \tau)_{, 1} .
\end{aligned}
$$

Substituting (68) and (69) into (67), we obtain

$$
\begin{array}{rl}
\Delta \Phi \geq 2 & 2 \delta \frac{\left(\rho_{, i j}\right)^{2}}{\rho^{2}}+\left(-2 n-8+\frac{2 n(1-\delta)}{n-1}\right) \\
& \times \frac{\left(\rho_{, 1}\right)^{2} \rho_{, 11}}{\rho^{3}}+2 R_{11} \frac{\left(\rho_{, 1}\right)^{2}}{\rho^{2}} \\
& +\left(\frac{n^{2}(1-\delta)}{2(n-1)}+2(n+3)\right) \frac{\left(\rho_{, 1}\right)^{4}}{\rho^{4}} \\
& +2 \frac{\rho_{, 1}}{\rho^{2}}(\rho \tau)_{, 1}-\frac{4 n-2-2 n \delta}{n-1} \Phi \tau+(1-\delta) \\
& \times\left(\frac{2 n}{n-1} \frac{\left(\rho_{, 11}\right)^{2}}{\rho^{2}}+4 \frac{\sum_{i>1}\left(\rho_{, 1 i}\right)^{2}}{\rho^{2}}\right. \\
& \left.-\frac{4}{n-1} \frac{\rho_{, 11}}{\rho} \tau+\frac{2}{n-1} \tau^{2}\right) .
\end{array}
$$

Note that

$$
\frac{\left(\rho_{, 11}\right)^{2}}{\rho^{2}}=\frac{1}{4} \sum \frac{\left(\Phi_{, i}\right)^{2}}{\Phi}-\frac{\sum_{i>1}\left(\rho_{, 1 i}\right)^{2}}{\rho^{2}}+2 \frac{\left(\rho_{, 1}\right)^{2} \rho_{, 11}}{\rho^{3}}-\frac{\left(\rho_{, 1}\right)^{4}}{\rho^{4}} .
$$


Then, (70) and (71) together give us

$$
\begin{array}{rl}
\Delta \Phi \geq 2 & 2 \delta \sum \frac{\left(\rho_{, i j}\right)^{2}}{\rho^{2}}+\frac{n(1-\delta)}{2(n-1)} \frac{\sum\left(\Phi_{, i}\right)^{2}}{\Phi} \\
& +\left(\frac{6 n(1-\delta)}{n-1}-2(n+4)\right) \frac{\left(\rho_{, 1}\right)^{2} \rho_{, 11}}{\rho^{3}}+2 R_{11} \frac{\left(\rho_{, 1}\right)^{2}}{\rho^{2}} \\
& +\left[\frac{\left(n^{2}-4 n\right)(1-\delta)}{2(n-1)}+2(n+3)\right] \frac{\left(\rho_{, 1}\right)^{4}}{\rho^{4}} \\
& +\frac{1-\delta}{n-1}\left(2 \tau^{2}-4 \frac{\rho_{, 11}}{\rho} \tau\right)-\frac{4 n-2-2 n \delta}{n-1} \Phi \tau \\
& +2 \frac{\rho_{, 1}}{\rho^{2}}(\rho \tau)_{, 1} .
\end{array}
$$

Using the Schwarz inequality gives

$$
2 \frac{\rho_{, 11}}{\rho} \tau \leq \frac{7}{3} \sum \frac{\left(\rho_{, i j}\right)^{2}}{\rho^{2}}+\frac{3}{7} \tau^{2}
$$

Using

$$
\frac{\left(\rho_{, 1}\right)^{2} \rho_{, 11}}{\rho^{3}}=\frac{1}{2} \Phi_{, i} \frac{\rho_{, i}}{\rho}+\Phi^{2},
$$

and choosing $\delta=7 /(3 n+4)$, we get

$$
\begin{aligned}
\Delta \Phi \geq & \frac{n(1-\delta)}{2(n-1)} \frac{\sum\left(\Phi_{, i}\right)^{2}}{\Phi}+\left(\frac{3 n(1-\delta)}{n-1}-(n+4)\right) \\
& \times \sum \Phi_{, i} \frac{\rho_{, i}}{\rho}+2 R_{11} \frac{\left(\rho_{, 1}\right)^{2}}{\rho^{2}} \\
& +\left[\frac{\left(n^{2}+8 n\right)(1-\delta)}{2(n-1)}-2\right] \Phi^{2}+\frac{8(1-\delta)}{7(n-1)} \tau^{2} \\
& -\frac{4 n-2-2 n \delta}{n-1} \Phi \tau+2 \frac{\rho_{, 1}}{\rho^{2}}(\rho \tau)_{, 1} .
\end{aligned}
$$

In the following, we will calculate the terms $R_{11}\left(\left(\rho_{, 1}\right)^{2} / \rho^{2}\right)$ and $\left(\rho_{, 1} / \rho^{2}\right)(\rho \tau)_{, 1}$. Note that (17) is invariant under an affine transformation of coordinates that preserved the origin. So, we can choose the coordinates $x_{1}, x_{2}, \ldots, x_{n}$ such that $f_{i j}(p)=\delta_{i j}$ and $\partial \rho / \partial x_{1}=\|\operatorname{grad} \rho\|(p)>0,\left(\partial \rho / \partial x_{i}\right)(p)=0$, for all $i>1$. From (19), we easily obtain

$$
\rho_{, i j}=\rho_{i j}+A_{i j 1} \rho_{, 1}=\frac{\rho_{, i} \rho_{, j}}{\rho}-A_{i j 1} \rho_{, 1}+\frac{A_{i j k} f_{k} \rho}{n+2} .
$$

Thus, we get

$$
\begin{gathered}
\Phi_{, i}=\frac{2 \rho_{, 1} \rho_{, 1 i}}{\rho^{2}}-2 \frac{\rho_{, i}\left(\rho_{, 1}\right)^{2}}{\rho^{3}}=-2 A_{i 11} \frac{\left(\rho_{, 1}\right)^{2}}{\rho^{2}}+2 \frac{\rho_{, 1} f_{k} A_{k i 1}}{(n+2) \rho} \\
\sum \Phi_{, i} \frac{\rho_{, i}}{\rho}=-2 A_{111} \frac{\left(\rho_{, 1}\right)^{3}}{\rho^{3}}+2 \frac{f_{k} A_{k 11}}{n+2} \frac{\left(\rho_{, 1}\right)^{2}}{\rho^{2}}
\end{gathered}
$$

By the same method, as deriving (69), we have

$$
\begin{aligned}
\sum\left(A_{m l 1}\right)^{2} \geq & \frac{n}{n-1} \sum\left(A_{i 11}\right)^{2}-\frac{2}{n-1} A_{111} \sum A_{i i 1} \\
& +\frac{1}{n-1}\left(\sum A_{i i 1}\right)^{2} .
\end{aligned}
$$

Note that $\sum A_{i i 1}=((n+2) / 2)\left(\rho_{1} / \rho\right)$. Therefore, by (14), (77), (78), and (79), we obtain

$$
\begin{aligned}
2 R_{11} \frac{\left(\rho_{, 1}\right)^{2}}{\rho^{2}}= & 2 \sum\left(A_{k j 1}\right)^{2} \frac{\left(\rho_{, 1}\right)^{2}}{\rho^{2}}-(n+2) A_{111} \frac{\left(\rho_{, 1}\right)^{3}}{\rho^{3}} \\
\geq & \frac{n}{2(n-1)} \frac{\sum\left(\Phi_{, i}-2\left(\rho_{, 1} f_{k} A_{k i 1} /(n+2) \rho\right)\right)^{2}}{\Phi} \\
& +\frac{(n+2)(n+1)}{2(n-1)} \sum \Phi_{, i} \frac{\rho_{, i}}{\rho} \\
& -\frac{n+1}{n-1} f_{k} A_{k 11} \frac{\left(\rho_{, 1}\right)^{2}}{\rho^{2}}+\frac{(n+2)^{2}}{2(n-1)} \Phi^{2} .
\end{aligned}
$$

On the other hand, we have

$$
2 \frac{\rho_{, 1}}{\rho^{2}}(\rho \tau)_{, 1}=2 \Phi \tau+\frac{1}{n+2} \sum A_{1 i k} f_{k} f_{i} \frac{\rho_{, 1}}{\rho}+\Phi
$$

Then, inserting (80) and (81) into (75), we get

$$
\begin{aligned}
\Delta \Phi \geq & \frac{2 n-n \delta}{2(n-1)} \sum \frac{\left(\Phi_{, i}\right)^{2}}{\Phi}-\frac{(n+2)(n-5)+6 n \delta}{2(n-1)} \\
& \times \sum \Phi_{, i} \frac{\rho_{, i}}{\rho}+\Phi+\frac{2(n+2)^{2}-\left(n^{2}+8 n\right) \delta}{2(n-1)} \Phi^{2} \\
& +\frac{8(1-\delta)}{7(n-1)} \tau^{2}-\frac{2 n(1-\delta)}{n-1} \Phi \tau+\frac{1}{n+2} \\
& \times \sum A_{1 i k} f_{k} f_{i} \frac{\rho_{, 1}}{\rho}-\frac{n+1}{n-1} \frac{\left(\rho_{, 1}\right)^{2}}{\rho^{2}} f_{k} A_{k 11} \\
& -\frac{2 n}{(n-1)(n+2)} \frac{\sum \Phi_{, i} f_{k} A_{k i 1}}{\sqrt{\Phi}}+\frac{2 n}{(n-1)(n+2)^{2}} \\
& \times \sum\left(f_{k} A_{k i 1}\right)^{2} .
\end{aligned}
$$

Using (77), we have

$$
\begin{gathered}
\frac{1}{n+2} \sum A_{1 i k} f_{k} f_{i} \frac{\rho_{, 1}}{\rho}-\frac{n+1}{n-1} \frac{\left(\rho_{, 1}\right)^{2}}{\rho^{2}} f_{k} A_{k 11} \\
=\frac{1}{2} f_{i} \Phi_{, i}-\frac{2}{n-1} \frac{\left(\rho_{, 1}\right)^{2}}{\rho^{2}} f_{k} A_{k 11} .
\end{gathered}
$$


One observes that the Schwarz inequality gives

$$
\begin{aligned}
& \frac{2 n}{(n-1)(n+2)} \frac{\sum \Phi_{, i} f_{k} A_{k i 1}}{\sqrt{\Phi}} \\
& \leq \frac{9 n}{8(n-1)} \sum \frac{\left(\Phi_{, i}\right)^{2}}{\Phi}+\frac{8 n}{9(n-1)(n+2)^{2}} \\
& \quad \times \sum\left(f_{k} A_{k i 1}\right)^{2}, \\
& \frac{2}{n-1} \frac{\left(\rho_{, 1}\right)^{2}}{\rho^{2}} f_{k} A_{k 11} \\
& \leq \frac{9(n+2)^{2}}{10 n(n-1)} \Phi^{2}+\frac{10 n}{9(n-1)(n+2)^{2}} \\
& \quad \times \sum\left(f_{k} A_{k i 1}\right)^{2}, \\
& 2 n \Phi \tau \leq \tau^{2}+n^{2} \Phi^{2} .
\end{aligned}
$$

Note that by (17) we have

$$
\begin{aligned}
\frac{1}{4} f^{i j} \Phi_{j} f_{i} & =\frac{n+2}{2} f^{i j} \Phi_{j}(\ln \rho)_{i}+\frac{1}{4} \Phi_{j} x_{j} \\
& =\frac{n+2}{2} f^{i j} \Phi_{j}(\ln \rho)_{i}+\frac{1}{4} f^{i j} \frac{\partial \Phi}{\partial x_{i}} \frac{\partial u}{\partial x_{j}}
\end{aligned}
$$

Then, inserting these estimates into (82) yields Proposition 6.

Proof of Corollary 8. Now, we will calculate the term $(\ln \rho)_{, i j k}$. In particular, if $f$ satisfies PDE (4), choose the coordinate $\left(x_{1}, x_{2}, \ldots, x_{n}\right)$ such that $f_{i j}(p)=\delta_{i j}$; then we have

$$
\begin{aligned}
(\ln \rho)_{, i j k}= & \frac{1}{n+2}\left(A_{i j k}+A_{i j k, p} f_{, p}\right)-(\ln \rho)_{, l} A_{i j k, l} \\
& +A_{i j l} A_{k l p}\left(3(\ln \rho)_{, p}-\frac{2}{n+2} f_{, p}\right) .
\end{aligned}
$$

Using (17), we have

$$
3(\ln \rho)_{, p}-\frac{2}{n+2} f_{, p}=-\frac{1}{n+2}(f+u)_{, p}+(\ln \rho)_{, p} .
$$

By the Young inequality and the Schwarz inequality, we have

$$
\begin{aligned}
& \frac{n+2}{n(n-1)} \sum A_{i j k} A_{i j l} A_{k l h}(\ln \rho)_{, h} \\
& \quad \leq \frac{1}{2} J^{2}+16 n^{2}(n-1)^{2}(n+2)^{4} \Phi^{2}, \\
& \frac{1}{n(n-1)} \sum A_{i j k} A_{i j l, k} f_{l} \\
& \quad=\frac{1}{2} \sum J_{, l} f_{, l}=\frac{1}{4}\langle\nabla J, \nabla(f+u)\rangle+\frac{n+2}{2}\langle\nabla J, \nabla \ln \rho\rangle,
\end{aligned}
$$

$$
\begin{aligned}
& \frac{n+2}{n(n-1)} \sum A_{i j k} A_{i j l, k}(\ln \rho)_{, l} \\
& \quad=\frac{n+2}{2} \sum J_{, i}(\ln \rho)_{, i} \\
& \leq \frac{1}{n(n-1)} \sum\left(A_{i j k, l}\right)^{2}+\frac{(n+2)^{2}}{4} J \Phi \\
& \leq \frac{1}{n(n-1)} \sum\left(A_{i j k, l}\right)^{2}+\frac{1}{4} J^{2}+\frac{(n+2)^{4}}{16} \Phi^{2}, \\
& \frac{1}{n(n-1)} \sum A_{i j k} A_{j i l} A_{k l p}(f+u)_{, p} \\
& \leq \sqrt{n(n-1)}\|\nabla(f+u)\| J^{3 / 2} .
\end{aligned}
$$

Thus, by inserting (88) into Lemma 7, we obtain Corollary 8.

\section{Conflict of Interests}

The authors declare that there is no conflict of interests regarding the publication of this paper.

\section{Acknowledgments}

The first author is supported by Grants (nos. 11101129 and 11201318) of NSFC. Part of this work was done when the first author was visiting the University of Washington and he thanks Professor Yu Yuan and the support of China Scholarship Council. The second author is supported by Grants (nos. U1304101 and 11171091) of NSFC and NSF of Henan Province (no. 132300410141).

\section{References}

[1] T. H. Colding and I. Minicozzi, "Generic mean curvature flow I: generic singularities," Annals of Mathematics, vol. 175, no. 2, pp. 755-833, 2012.

[2] K. Ecker and G. Huisken, "Mean curvature evolution of entire graphs," Annals of Mathematics, vol. 130, no. 3, pp. 453-471, 1989.

[3] G. Huisken, "Asymptotic behavior for singularities of the mean curvature flow," Journal of Differential Geometry, vol. 31, no. 1, pp. 285-299, 1990.

[4] G. Huisken, "Local and global behaviour of hypersurfaces moving by mean curvature," in Differential Geometry: Partial Differential Equations on Manifolds, vol. 54 of Proceedings of Symposia in Pure Mathematics, American Mathematical Society, 1993.

[5] K. Smoczyk, "Self-shrinkers of the mean curvature flow in arbitrary codimension," International Mathematics Research Notices, no. 48, pp. 2983-3004, 2005.

[6] L. Wang, "A Bernstein type theorem for self-similar shrinkers," Geometriae Dedicata, vol. 151, pp. 297-303, 2011.

[7] H. D. Cao and H. Li, "A gap theorem for self-shrinkers of the mean curvature flow in arbitrary codimension," Calculus of Variations and Partial Differential Equations, vol. 46, no. 3-4, pp. 879-889, 2013. 
[8] A. Chau, J. Chen, and Y. Yuan, "Rigidity of entire self-shrinking solutions to curvature flows," Journal für die Reine und Angewandte Mathematik, vol. 664, pp. 229-239, 2012.

[9] Q. Ding and Z. Z. Wang, On the self-shrinking systems in arbitrary codimension spaces, http://arxiv.org/abs/1012.0429.

[10] Q. Ding and Y. L. Xin, "The Rigidity theorems for Lagrangian self-shrinkers," Journal für die Reine und Angewandte Mathematik, 2012.

[11] K. Ecker, "Interior estimates and longtime solutions for mean curvature flow of noncompact spacelike hypersurfaces in Minkowski space," Journal of Differential Geometry, vol. 46, no. 3, pp. 481-498, 1997.

[12] R. Huang and Z. Wang, "On the entire self-shrinking solutions to Lagrangian mean curvature flow," Calculus of Variations and Partial Differential Equations, vol. 41, no. 3-4, pp. 321-339, 2011.

[13] Y. L. Xin, "Mean curvature flow with bounded Gauss image," Results in Mathematics, vol. 59, no. 3-4, pp. 415-436, 2011.

[14] Y. L. Xin, Minimal Submanifolds and Related Topics, World Scientific Publishing, 2003.

[15] A.-M. Li, R. Xu, U. Simon, and F. Jia, Affine Bernstein Problems and Monge-Ampère Equations, World Scientific, Singapore, 2010.

[16] A. M. Li and R. W. Xu, "A rigidity theorem for an affine KählerRicci flat graph," Results in Mathematics, vol. 56, no. 1-4, pp. 141164, 2009.

[17] A.-M. Li and R. Xu, "A cubic form differential inequality with applications to affine Kähler-Ricci flat manifolds," Results in Mathematics, vol. 54, no. 3-4, pp. 329-340, 2009.

[18] R. W. Xu and R. L. Huang, "On the rigidity theorems for Lagrangian translating solitons in pseudo-Euclidean space I," Acta Mathematica Sinica (English Series), vol. 29, no. 7, pp. 13691380, 2013.

[19] A. V. Pogorelov, The Minkowski Multidimensional Problem, John Wiley \& Sons, New York, NY, USA, 1978.

[20] E. Calabi, "Improper affine hyperspheres of convex type and a generalization of a theorem by K. Jörgens," The Michigan Mathematical Journal, vol. 5, pp. 105-126, 1958. 


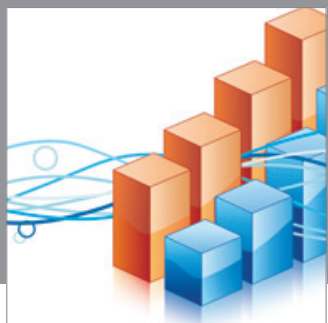

Advances in

Operations Research

mansans

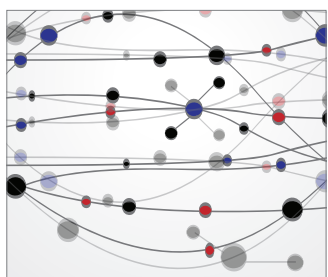

The Scientific World Journal
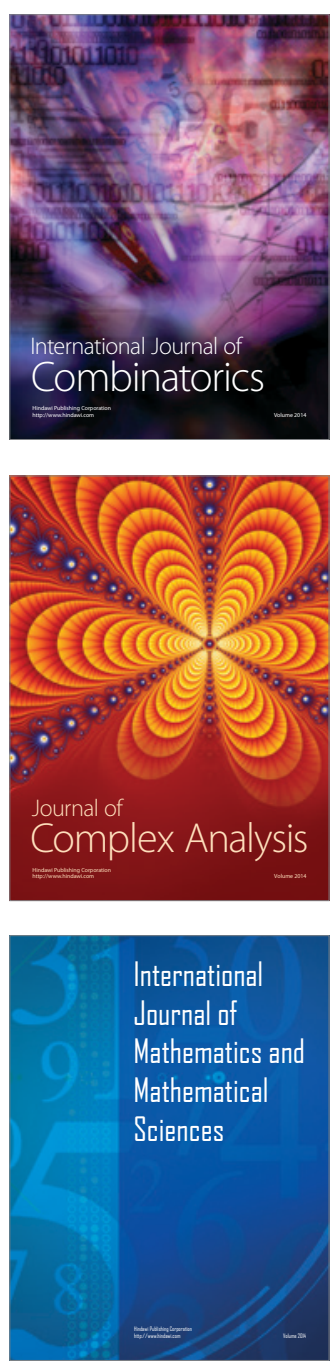
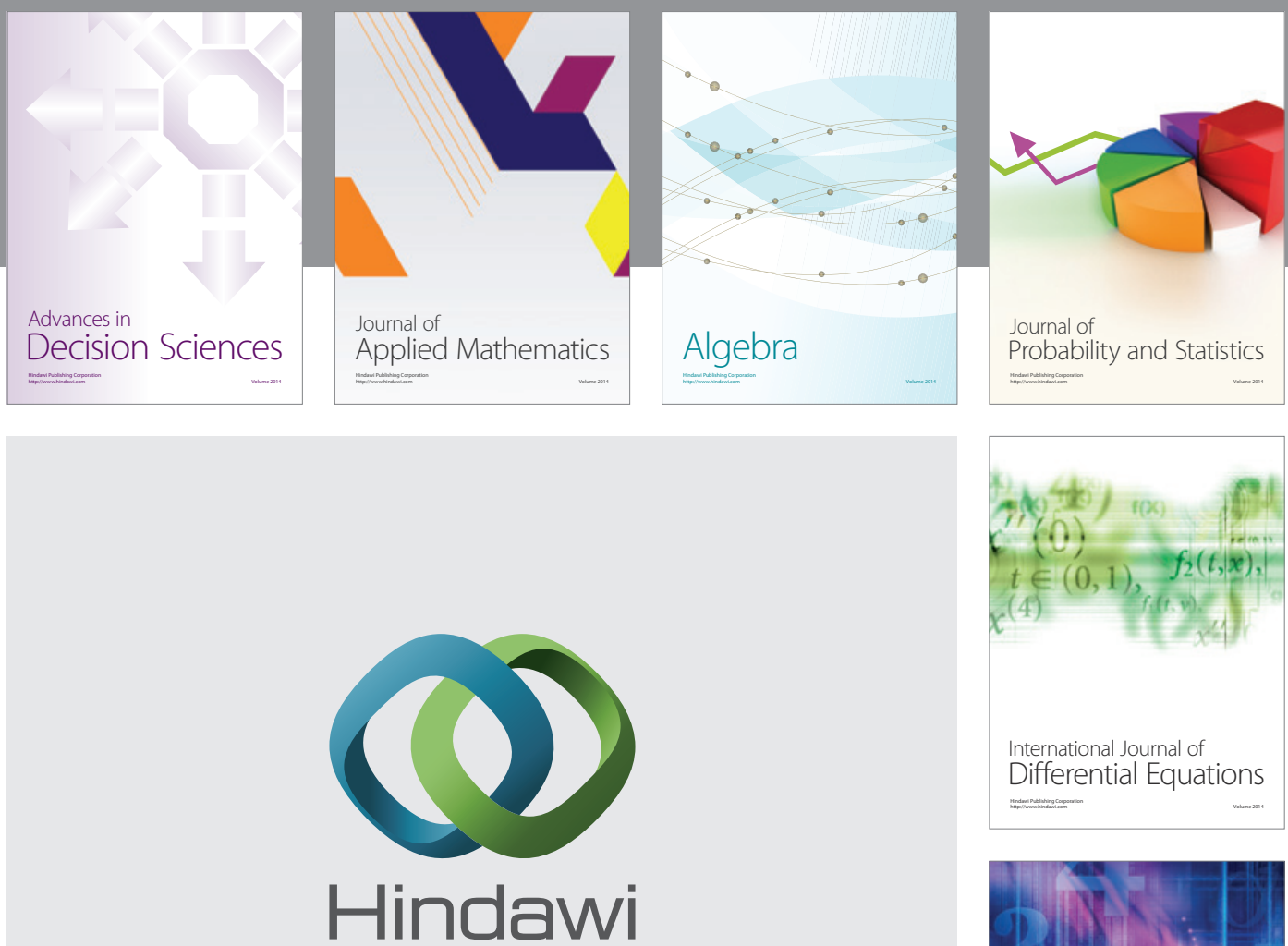

Submit your manuscripts at http://www.hindawi.com
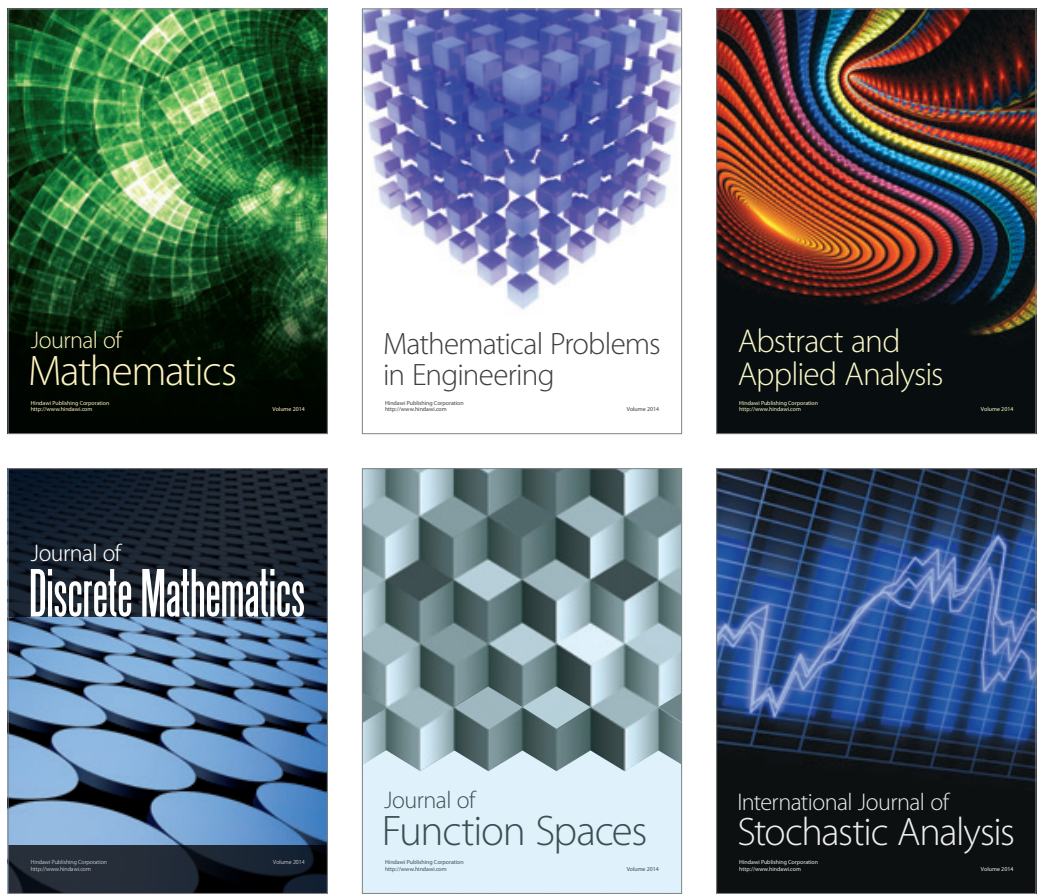

Journal of

Function Spaces

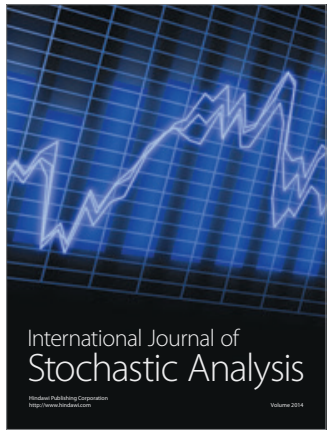

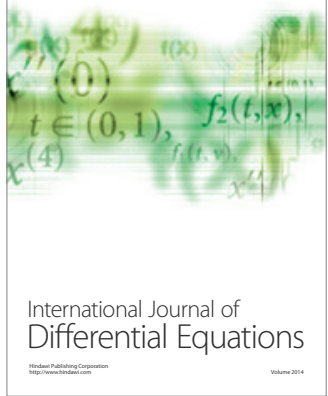
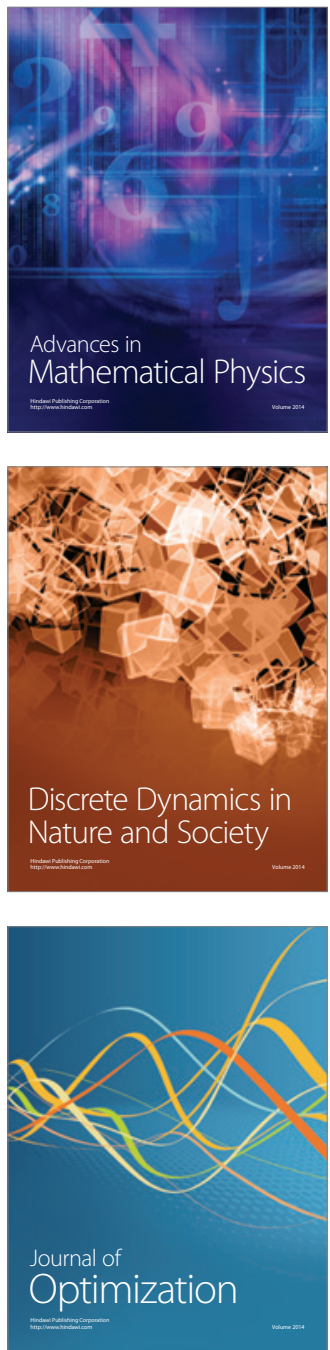